\title{
Technical note \\ Significance and determination of fraction of non-separable particles of impurities in water purification
}

\author{
P Polasek* \\ Water and Wastewater Treatment Consultant, PO Box 65138, Erasmusrand 0165, South Africa
}

\begin{abstract}
The method to determine the quantity of non-separable particles in water, together with the calculations for the determination of the conditions of centrifugation under which the quantity of the non-separable particles can be determined, are dealt with in this paper. It is shown that for the determination of the quantity of non-separable particles it is beneficial to replace sedimentation in a gravity field with sedimentation in a centrifugal field. Reduction in the quantity of non-separable particles is characterised by the degree of destabilisation $\delta$, which is proportional to the efficiency of the purification process. The development of separable aggregates is characterised by the degree of aggregation $\alpha_{A}$ which is proportional to the aggregation and separation efficiency of the system. The degree of destabilisation $\delta$ corresponds to the collision frequency factor $\alpha_{P}$ in the Smoluchowski equation for the perikinetic coagulation. The degree of aggregation $\alpha_{A}$ corresponds to the collision frequency factor $\alpha_{0}$ in the Smoluchowski equation for orthokinetic coagulation.
\end{abstract}

Keywords: non-separable particles, number of non-separable particles, degree of destabilisation, degree of aggregation, centrifugation, collision frequency factors

\section{INTRODUCTION}

The impurities which chemical water purification seeks to remove are present in water in the form of aggregately and kinetically stable dispersions. The prerequisite for removal of these particles is the removal of their aggregate stability, referred to as particle destabilisation. Particle destabilisation is achieved by addition of a destabilisation agent such as hydrolysing aluminium or iron salt. With a sufficient dosage of destabilisation agent, the energy barrier of particles is reduced to such an extent that the particles are brought into such close proximity that the influence of adhesion forces predominates and the particles combine into readily settleable aggregates.

The total number of particles of every pollutant in water is the sum of its two different particle size-fractions, namely:

- The separable (sedimentable) particles which can be removed from water by a suitable separation process such as sedimentation, flotation, centrifugation or deep bed filtration

- The non-separable particles which cannot be removed from water by any common separation method without appropriate chemical treatment

For the purpose of simplification of measurement, the number of particles is expressed by the concentration of determinant. The total number/concentration of particles on any pollutant is expressed as follows:

$$
N_{o}=N_{S}+N_{N S} \quad C_{O}=C_{S}+C_{N S}
$$

\footnotetext{
To whom all correspondence should be addressed.

용 +27 82 833-4330; e-mail: polasek@mweb.co.za

Received 21 January 2013; accepted in revised form 6 December 2013.
}

where:

$N_{O}\left(C_{0}\right)$ equals the total number (concentration) of particles of any determinant

$N_{S}\left(C_{S}\right)$ is the fraction of the total number (concentration) of particles which is produced by the separable particles $N_{N S}\left(C_{N S}\right)$ is the remaining fraction of the total number (concentration) of particles which is produced by the nonseparable particles.

Due to the variety of mechanisms that may be engaged in the transformation of particles of impurities present in water into separable aggregates using a hydrolysing coagulant, the term coagulation does not reflect all of the partial processes taking place during the transformation of colloidal impurities into readily separable flocs. It is more precise to call this process 'aggregation' and to call the flocs formed 'aggregates', as well as to call the hydrolysing coagulant 'destabilisation agent'. For this reason the terms aggregation, aggregates and destabilisation agent are used in this paper.

The objectives of this paper are to highlight the significance of the non-separable particles with regard to the quality of purified water and to provide a guide for determining the number of non-separable particles of impurities in water. Evidence of the impact of non-separable particles on the quality of purified water is also documented.

\section{The kinetics of formation of separable aggregates}

Particle aggregation proceeds gradually with random collisions between the destabilised particles producing kinetically unstable aggregates. The kinetics of the aggregation process is described by basic relationships developed by Smoluchowski $(1917,1918)$ as follows: 
- For the perikinetic coagulation:

$$
b_{i j}=4 \pi D_{i j} N_{i} N_{j} \alpha_{P}
$$

- And for the orthokinetic coagulation:

$$
b_{i j}=\frac{4}{3} G R_{i j}^{3} N_{i} N_{j} \alpha_{O}
$$

It follows from the above relationships that the kinetics of the process of perikinetic coagulation, i.e., the collision frequency factor $b_{i j}$, is influenced by the number of particles $N_{i}$ and $N_{i}$ and diffusion coefficient $D_{i j}$. For orthokinetic coagulation $b_{i j}$ is influenced by the number of particles $N_{i}$ and $N_{i}$, effective radius of particles $R_{i j}$ and velocity gradient $G$. In both cases $b_{i j}$ is also influenced by chemical factors characterised by the collision efficiency factors $\alpha_{p}$ and $\alpha_{O}$.

The collision efficiency factor $\alpha_{P}$ characterises the influence of chemical factors on the speed of the destabilisation process. It is defined as a ratio of the particle collisions which resulted in the combining of particles to the total number of collisions under the conditions of Brownian motion. Its value varies between 0 and 1 .

The collision efficiency factor $\alpha_{O}$ characterises the development of aggregates. It is defined as a ratio of particle collisions which resulted in aggregation to the total number of collisions owing to the differential movement of volumes of water, which is characterised by the velocity gradient. Its value varies between 0 and 1 .

\section{Degree of destabilisation}

The number of non-separable particles remaining in the system characterises the efficiency of the destabilisation process. This efficiency may be evaluated by the degree of destabilisation $(\delta)$ (Polasek, 1980). The degree of destabilisation $(\delta)$ is defined as the ratio of the number of the particles which destabilised during the purification process $\left(N_{D}\right)$ to the initial total number of the aggregately-stable particles $\left(N_{S}\right)$ present in the system as follows:

$$
\delta=\frac{N_{D}}{N_{S}}=\frac{C_{D}}{C_{S}}=\frac{C_{O} F-C F}{C_{O} F}
$$

The degree of destabilisation $\delta$ is calculated from the initial concentration of the non-separable particles $\left(C_{O} F\right)$ measured in the raw water and the concentration of the non-separable (non-destabilised) particles $(C F)$ remaining in the water at the point of testing, i.e., from the concentration of particles which has destabilised and aggregated during the purification process $\left(C_{D}=C_{O} F-C F\right)$ to the initial concentration of the non-separable particles $C_{O} F$ in the system according to Eq. (4). The initial concentration of all aggregately-stable particles $C_{O} F$ is the concentration of non-separable particles measured in the centrifuged sample of the raw water. In the case of the cation of the destabilisation agent it is measured in a sample of water after dispersion of the agent.

The degree of destabilisation $\delta$ is influenced by the state of the system at the moment of addition of the destabilisation agent. The state of the system is characterised by the composition of the impurities, the reaction $\mathrm{pH}$, the water temperature, the destabilisation agent used, its dosage, and efficiency of its dispersion and homogenisation with water. The value of the degree of destabilisation $\delta$ varies between 0 and $1 ; \delta=0$ corresponds to the state immediately before addition of the destabilisation agent to the water when the destabilisation process has not yet commenced and when none of the non-separable particles has been destabilised; $\delta=1$ corresponds to the state when the destabilisation process is completed and all particles of the impurity are destabilised. When $\delta=0$ none of the initial aggregately-stable particles are capable of aggregation and, therefore, are not removable from the water. When $\delta=1$ all of the aggregately-stable particles are destabilised and capable of aggregation.

In principle, the degree of destabilisation is a measure of the attainable treatability of the water. This provides a very rapid method, which has very good reproducibility of results.

Based on definitions of both the collision efficiency factor $\alpha_{P}$ in the Smoluchovski Eq. for perikinetic coagulation and the degree of destabilisation $\delta$, it is evident that the value of $\delta$ is enumerating the factor $\alpha_{p}$.

\section{Degree of aggregation}

During the process of particle aggregation substantial changes in the number and size of aggregates occur. These changes can be utilised for the evaluation of performance efficiency of the individual unit operations and the waterworks in its entirety.

The efficiency of the aggregation process can be evaluated by the degree of aggregation $\alpha_{A}$ (Hereit et al., 1977, 1980; Mutl and Polasek, 2002). It is defined as the ratio of the number of separable particles $N_{A}$ to the total number of all particles $N_{O}$ present in the system as follows:

$$
\alpha_{A}=\frac{N_{A}}{N_{O}}=\frac{C_{A}}{C_{O}}=\frac{C_{O}-C_{E} F}{C_{O}}
$$

The degree of aggregation $\alpha_{A}$ is calculated from the concentration $C_{O}$ of the monitored determinant measured in the raw water, from which the remaining concentration of non-separable particles measured at the point of investigation $C_{E} F$ is deducted to give the total initial concentration of determinant $C_{0}$.

The value of the degree of aggregation varies between $\alpha_{A}=0$ (none of the particles in the system are separable) and $\alpha_{A}=1$ (all particles in the system are separable). It follows from the theory of the kinetics of the aggregation process that the number of particles in the system decreases and the degree of aggregation $\alpha_{A}$ increases as the aggregation process progresses. The higher the value of $\alpha_{A}$ the higher is the aggregation efficiency attained by the system.

When the initial concentration of a determinant $C_{O}$ is measured in raw water, or, in the case of a destabilisation agent, after its dispersion and homogenisation with raw water in the dosed water, and concentration $C_{E} F$ is measured in the centrifuged sample taken at the outlets of the individual unit operations of the works, then the degree of aggregation $\alpha_{A}$ characterises the progress in the aggregation of particles achieved at such testing point.

As with the degree of destabilisation $\delta$, it can be inferred that the value of the degree of aggregation $\alpha_{A}$ enumerates the collision efficiency factor $\alpha_{O}$ in the Smoluchovski Eq. for orthokinetic coagulation.

\section{Determination of non-separable portion of particles}

The determination of the number of non-destabilised particles presents challenges. The diameter of the colloidal particles of impurities without an adsorbed destabilisation agent is of the order of $10^{-8} \mathrm{~m}$. The particle diameter with a partly adsorbed destabilisation agent, which does not enable sufficient destabilisation, is of the order of $10^{-7} \mathrm{~m}$. These particles move by 
perikinetic motion. They are kinetically stable and cannot be removed by sedimentation.

The aggregately-stable particles of impurities are of the analytical and colloidal degree of dispersion. The size of analytical dispersions is limited to the order of $d_{P} \leq 10^{-8} \mathrm{~m}$ and colloidal dispersions $10^{-8} \leq d_{P} \leq 10^{-6} \mathrm{~m}$. The number (concentration) of non-separable particles of impurities is determined in a sample of water from which all separable particles, including aggregates for which the diameter increased to $d_{A} \geq 1.5^{\star} 10^{-7} \mathrm{~m}$ due to aggregation, are removed. The removal of these particles by sedimentation in a gravity field would require an extremely long time period, as their calculated sedimentation velocity is of the order of $10^{-9} \mathrm{~m} \cdot \mathrm{s}^{-1}$. In addition, the sedimentation process is distorted by many factors acting upon the particles, such as thermal and density currents, concentration, vertical aggregation and chemical reactions. Therefore the actual time taken will be much longer than that derived from the calculated sedimentation velocity, if the smallest particles settle at all.

In view of the foregoing, it is advantageous to replace sedimentation in a gravity field by sedimentation in a centrifugal field. The distorting factors adversely affecting sedimentation in a gravity field are minimised under the influence of centrifugal forces because sedimentation takes place over a short distance and over a short time period.

\section{Calculation of conditions of centrifugation}

The conditions of centrifugation can be derived from the relationships defining sedimentation velocity of a spherical particle in a gravity field $u$ :

$$
u_{s}=\frac{g d^{2}\left(\rho_{P}-\rho_{W}\right)}{18 \mu}
$$

where:

$d$ is diameter of spherical particle,

$\rho_{P}$ is density of particles of impurities

$\rho_{W}$ is density of water

$\mu$ is absolute viscosity of water

By substitution of the gravity acceleration constant $g$ with the centrifugation acceleration constant $a$ :

$$
a=\omega^{2} R=4 \pi^{2} n^{2} R=\frac{\pi^{2} R P M^{2} R}{900}
$$

where:

$\omega$ is angular velocity

$n$ is centrifuge speed (revolutions per second)

$R$ is radius of centrifuge

$R P M$ is revolutions per minute

Angular velocity is given by:

$$
\omega=2 \pi n=\frac{\pi R P M}{30}
$$

The sedimentation velocity of a spherical particle in the centrifugal field and in laminar movement is determined by the relationship:

$$
u_{C}=\frac{a d^{2}\left(\rho_{P}-\rho_{W}\right)}{18 \mu}=\frac{\pi^{2} R P M^{2} d^{2} R\left(\rho_{P}-\rho_{W}\right)}{16,200 \mu}
$$

The Reynolds number is calculated as follows:

$$
\operatorname{Re}=\frac{d u_{C} \rho_{W}}{\mu}
$$

where:

$$
u_{C} \text { is sedimentation velocity under centrifugation }
$$

The time required for sedimentation of particles in a centrifugal field and in laminar flow is calculated as follows (Heinrich et al., 1970):

$$
d T=\frac{1}{k} \frac{d R}{R} \quad k=\frac{2}{9} \frac{\pi^{2} n^{2} d^{2}\left(\rho_{P}-\rho_{W}\right)}{\mu}
$$

After integration and substitution of Eq. (12) the time of centrifugation $T_{C}$ is calculated as follows:

$$
\begin{aligned}
T_{C} & =\frac{9 \mu}{2 \pi^{2} d_{P}^{2} n^{2}\left(\rho_{P}-\rho_{W}\right)} \ln \frac{R_{2}}{R_{1}} \\
& =\frac{1}{4 \pi^{2} n^{2} u_{C}} 2.3 \log \frac{R_{2}}{R_{1}}
\end{aligned}
$$

where:

$$
\begin{aligned}
& d_{P} \text { is diameter of the smallest particle to settle } \\
& R_{2} \text { is distance between the bottom of the container and } \\
& \text { the axis of centrifuge } \\
& R_{1} \text { is distance between water level in the centrifuge } \\
& \text { container and the axis of centrifuge }
\end{aligned}
$$

Table 1 shows the times of centrifugation for three basic types of impurities commonly found in water. These times are calculated for the removal of particles down to two sizes, namely $d_{P}=1 \mathrm{E}-06 \mathrm{~m}$ and $d_{P}=1.5 \mathrm{E}-07 \mathrm{~m}$. These times of centrifugation were calculated according to Eq. 12 for different water temperatures using Labofuge 1 laboratory centrifuge with swinging rotor, effective glass container capacity of $80 \mathrm{~m} \ell$ and rotation speed of $n=4200 \mathrm{~min}^{-1}$. The first particle size, $d_{P}=1 \mathrm{E}-06 \mathrm{~m}$, determines the upper size limit of colloidal particles. Removal of these particles is achieved within a range of shorter times of centrifugation, between $T_{C}=1.2$ and $13.0 \mathrm{~min}$, depending on the type of impurity and water temperature. The second particle size, $d_{P}=1.5 \mathrm{E}-07 \mathrm{~m}$, is considered to be the average size for the non-separable particles found in water. Removal of these particles is achieved within a range of longer times of centrifugation, between $T_{C}=57$ and $597 \mathrm{~min}$, depending on the type of impurity and water temperature.

Practical experience showed that for evaluating performance efficiency of each unit operation the determination of non-separable particles within the range of colloidal particles, i.e., the shorter time of centrifugation of about $10 \mathrm{~min}$, is satisfactory. When evaluating the efficiency of a filtration plant the quantity of non-separable particles remaining in the filtrate is usually slightly lower than that determined in the centrate produced by the shorter $T_{C}$. This is because some of the particles smaller than $d_{P}=1 \mathrm{E}-06 \mathrm{~m}$ are removed by filtration.

The time of centrifugation can also be experimentally determined for a selected type of centrifuge and speed of centrifugation. The samples of water dosed with destabilisation agent are centrifuged over different times and in the samples of the centrifuged water the selected determinant is analysed. Table 2 shows the time of centrifugation versus concentration of cation of destabilisation agent (Al). The procedure for optimisation of centrifugation time is evident from Table 2. The lowest concentration of $\mathrm{Al}$ obtained in the shortest time corresponds to the shortest time of centrifugation which is required for the determination of non-separable particles produced by common impurities. The results obtained show that the shortest time of centrifugation required to remove separable particles of these impurities is about $140 \mathrm{~min}$. This 


\section{TABLE 1}

Time of centrifugation $T_{C}$ required for determination of non-separable particles of two basic particle sizes $d_{p}$ using Labofuge 1 centrifuge running at $n=4200 \mathrm{~min}^{-1}$ for various types of impurities and different water temperatures

\begin{tabular}{|c|c|c|c|c|c|c|}
\hline \multirow[t]{2}{*}{ Type of impurity } & $t$ & $\rho_{0}$ & $d_{p}$ & $T_{c}$ & $d_{p}$ & $\mathrm{~T}_{\mathrm{c}}$ \\
\hline & $\left({ }^{\circ} \mathrm{C}\right)$ & $\left(\mathrm{kg} \cdot \mathrm{m}^{-3}\right)$ & (m) & $(\mathrm{min})$ & $(\mathrm{m})$ & $(\mathrm{min})$ \\
\hline Common impurities & \multirow[t]{3}{*}{4} & 1650 & $1 \mathrm{E}-06$ & 4.1 & $1.5 \mathrm{E}-07$ & 184 \\
\hline Clay & & 2500 & $1 \mathrm{E}-06$ & 1.8 & $1.5 \mathrm{E}-07$ & 80 \\
\hline Humic substances & & 1200 & $1 \mathrm{E}-06$ & 13.4 & $1.5 \mathrm{E}-07$ & 597 \\
\hline Common impurities & \multirow[t]{3}{*}{10} & 1650 & $1 \mathrm{E}-06$ & 3.6 & $1.5 \mathrm{E}-07$ & 159 \\
\hline Clay & & 2500 & $1 \mathrm{E}-06$ & 1.5 & $1.5 \mathrm{E}-07$ & 69 \\
\hline Humic substances & & 1200 & $1 \mathrm{E}-06$ & 11.6 & $1.5 \mathrm{E}-07$ & 515 \\
\hline Common impurities & \multirow[t]{3}{*}{15} & 1650 & $1 \mathrm{E}-06$ & 3.1 & $1.5 \mathrm{E}-07$ & 138 \\
\hline Clay & & 2500 & $1 \mathrm{E}-06$ & 1.3 & $1.5 \mathrm{E}-07$ & 60 \\
\hline Humic substances & & 1200 & $1 \mathrm{E}-06$ & 10.1 & $1.5 \mathrm{E}-07$ & 448 \\
\hline Common impurities & \multirow[t]{3}{*}{20} & 1650 & $1 \mathrm{E}-06$ & 3.0 & $1.5 \mathrm{E}-07$ & 131 \\
\hline Clay & & 2500 & $1 \mathrm{E}-06$ & 1.3 & $1.5 \mathrm{E}-07$ & 57 \\
\hline Humic substances & & 1200 & $1 \mathrm{E}-06$ & 9.6 & $1.5 \mathrm{E}-07$ & 425 \\
\hline
\end{tabular}

\section{TABLE 2}

Experimental determination of centrifugation conditions for determining non-separable particles using Labofuge 1 centrifuge $\left(t=16^{\circ} \mathrm{C}, n=4200 \mathrm{~min}^{-1}\right)$

\begin{tabular}{|l|c|c|c|c|c|c|c|c|}
\hline Time of centrifugation & $(\mathrm{min})$ & 5 & 15 & 30 & 60 & 120 & 180 & 240 \\
\hline Al in dosed raw water sample & $\left(\mathrm{mg} \cdot \ell^{-1}\right)$ & 1.18 & 1.20 & 1.21 & 1.18 & 1.20 & 1.18 & 1.18 \\
\hline Al in centrifuged water sample & $\left(\mathrm{mg} \cdot \ell^{-1}\right)$ & 0.46 & 0.26 & 0.145 & 0.110 & 0.105 & 0.103 & 0.103 \\
\hline$\delta$ & $(-)$ & 0.61 & 0.78 & 0.88 & 0.907 & 0.913 & 0.913 & 0.913 \\
\hline
\end{tabular}

corresponds reasonably well with the time of centrifugation (138 min) for common impurities calculated in Table 1. The same Labofuge 1 laboratory centrifuge with swinging rotor and with effective glass container capacity of $80 \mathrm{~m} \ell$ and rotation speed of $n=4200 \mathrm{~min}^{-1}$ was used.

\section{Significance of the impact of non-separable particles on the quality of purified water}

The process of chemical purification of water is aimed at converting the non-separable particles to separable ones and lowering the degree of dispersion of the particles of impurities in the system. The degree of dispersion is defined as the inverse value of the linear dimension of particles. The higher the degree of dispersion the smaller is the particle size and vice versa. Since the kinetic stability is defined as the resistance of particles against the influence of a force field, the particles that are not susceptible to the influence of a force field are not removable by sedimentation and are considered to be kinetically stable. An objective of water purification is to attain the transformation of kinetically stable particles to kinetically unstable aggregates.

Only those particles of impurities which are sufficiently destabilised and have aggregated into separable aggregates can be removed from the water. This means that the non-separable particles are comprised of initial stable particles, destabilised but non-aggregated particles, particles which are aggregated to the lowest order of magnitude and particles of destabilisation agent which did not enter into the destabilisation reactions. The destabilisation of the particles takes place immediately after adsorption of the destabilisation agent onto the surface of the particles. It follows that the quantity of the non-separable particles does not change much in the subsequent processes of aggregate formation and separation provided that the destabilisation process takes place under optimised reaction conditions. This is because the removal of the stable particles remaining in the system can only be effected by additional mechanisms such as sorption to the surface of aggregates, particle enmeshment and aggregation by forcibly overpowering the energy barrier which has not been sufficiently reduced. The remaining quantity of the aggregately-stable particles that were not sufficiently destabilised, and therefore could not be aggregated, determine the attainable treatability of the water. Therefore, the aim of the operator should always be to operate the waterworks under the optimised reaction conditions.

Determinants for which the concentration changes during the purification process are used for the evaluation of the treatability of water. Determinants most often used are: cation of destabilisation agent ( $\mathrm{Al}$ or $\mathrm{Fe}$ ), DOC, TOC or $\mathrm{COD}_{\mathrm{Mn}}$, colour, turbidity, alkalinity, content of suspended solids, and type and count of micro-organisms.

The practicality and usefulness of the degree of destabilisation $\delta$ as a tool for the evaluation of the quality of purified water and for the comparison of waterworks performance efficiency, as well as the identification of bottlenecks inherent in the plant design, is illustrated in Tables 3 and 4.

The frmation of separable aggregates by means of an organic cationic polyelectrolyte (CPE) takes place by a different mechanism. CPE, also referred to as polymeric coagulant, is either a pure cationic polymer or a blend of a cationic polymer, usually with aluminium chlorohydrate or polyaluminium chloride. The formation of aggregates by means of a cationic polymer takes place directly by bridging of particles of impurities without their aggregate destabilisation. In the case of polymeric coagulant some aggregate destabilisation of particles may occur in addition to bridging of particles of impurities. The mechanism of formation of aggregates is dependent on the 


\begin{tabular}{|l|c|c|}
\hline \multicolumn{3}{|c|}{$\begin{array}{c}\text { TABLE 3 } \\
\text { The effect of different coagulation agents on } \\
\text { waterworks performance efficiency evaluated } \\
\text { by turbidity (NTU) measurement } \\
\text { (From: Polasek, 2011) }\end{array}$} \\
\hline Parameters & $\begin{array}{c}\text { Engcobo WTW: } \\
\text { Cationic } \\
\text { polyelectrolyte } \\
\text { (Zetafloc 2350) }\end{array}$ & $\begin{array}{c}\text { Duivenhoks WTW: } \\
\text { Aluminium } \\
\text { sulphate }\end{array}$ \\
\hline Raw water: & 15.9 & 12.5 \\
\hline $\mathrm{C}_{0}$ & 1.80 & 1.80 \\
\hline $\mathrm{C}_{0} \mathrm{~F}$ & 1.79 & 0.17 \\
\hline Purified (filtered) water: & 0.76 & 0.11 \\
\hline $\mathrm{C}_{\mathrm{F}}$ & 1.03 & 0.06 \\
\hline $\mathrm{C}_{\mathrm{F}} \mathrm{F}$ & 0.58 & 0.94 \\
\hline$\Delta \mathrm{C}_{\mathrm{F}}$ & 0.952 & 0.991 \\
\hline$\delta$ & & \\
\hline$\alpha_{\mathrm{A}}$ & & \\
\hline
\end{tabular}

\begin{tabular}{|c|c|c|c|c|c|c|c|c|}
\hline \multicolumn{9}{|c|}{$\begin{array}{l}\text { TABLE } 4 \\
\begin{array}{l}\text { Comparison of performance efficiency of different type of clarifiers installed in the Bethlehem Waterworks } \\
\text { (From: Polasek and Van Duuren, 1981) }\end{array}\end{array}$} \\
\hline \multirow[t]{3}{*}{ Type of clarifier } & \multicolumn{2}{|c|}{ Dosing rate } & \multirow{2}{*}{$\begin{array}{l}\text { Upflow } \\
\text { velocity } \\
\left(m \cdot h^{-1}\right)\end{array}$} & \multirow[t]{2}{*}{ Point of measurement } & \multicolumn{2}{|c|}{ Turbidity } & \multicolumn{2}{|c|}{ Degree of: } \\
\hline & $\begin{array}{l}\text { Aluminium } \\
\text { sulphate } \\
\left(\mathrm{mg} \cdot \ell^{-1}\right)\end{array}$ & $\begin{array}{l}\text { SF-A110 } \\
\left(\mathrm{mg} \cdot \ell^{\mathrm{l}^{-1}}\right)\end{array}$ & & & $\begin{array}{c}\mathrm{C}_{\mathrm{o}} \\
\text { (NTU) }\end{array}$ & $\begin{array}{l}\mathrm{C}_{\mathrm{E}} \mathrm{F} \\
\text { (NTU) }\end{array}$ & $\begin{array}{c}\text { Destabil- } \\
\text { isation } \\
\Delta \\
(-) \\
\end{array}$ & $\begin{array}{c}\text { Aggre- } \\
\text { gation } \\
\boldsymbol{a}_{\mathrm{A}} \\
(-) \\
\end{array}$ \\
\hline & & & & Raw water & 102 & 54 & - & - \\
\hline \multirow{3}{*}{$\begin{array}{l}\text { Pretreator } \\
\text { (retention time of about } \\
3.5 \mathrm{~h} \text { ) }\end{array}$} & \multirow{3}{*}{60} & \multirow{3}{*}{0} & \multirow{3}{*}{1.5} & Flocculation chamber outlet & 102 & 4.9 & 0.909 & 0.952 \\
\hline & & & & Hydraulic jump & 102 & 4.0 & 0.926 & 0.960 \\
\hline & & & & Clarifier outlet & 7.1 & 1.5 & 0.972 & 0.985 \\
\hline \multirow{2}{*}{$\begin{array}{l}\text { Clariflocculator (reten- } \\
\text { tion time of about } 4 \mathrm{~h} \text { ) }\end{array}$} & \multirow{2}{*}{60} & \multirow{2}{*}{0} & \multirow{2}{*}{1.2} & Flocculation chamber outlet & 102 & 4.9 & 0.909 & 0.952 \\
\hline & & & & Clarifier outlet & 7.2 & 2.9 & 0.946 & 0.972 \\
\hline \multirow{2}{*}{$\begin{array}{l}\text { HR clarifier (retention } \\
\text { time of about } 20 \mathrm{~min} \text { ) }\end{array}$} & \multirow{2}{*}{52} & \multirow{2}{*}{0.172} & \multirow{2}{*}{15.5} & Flocculation chamber outlet & 102 & 1.7 & 0.969 & 0.983 \\
\hline & & & & Clarifier outlet & 7.0 & 1.4 & 0.974 & 0.986 \\
\hline
\end{tabular}

composition of polymeric coagulant. As a result, the performance efficiency of CPE for the removal of hydrophobic-type impurities such as cation of destabilisation agent and turbidity, and especially hydrophilic-type impurities such as organic pollution (natural organic matter - NOM), was found to be relatively limited (Polasek and Mutl, 2002). In view of this, CPE should only be used for the purification of raw waters with low NOM. However, the adverse side-effects of CPE on the performance of filtration plants preclude the use of CPE, even for raw water with low NOM. Therefore, the use of CPE is not considered to be suitable for the purification of water in any circumstances (Polasek and Mutl, 2002). This is also clearly evident from comparison of results in Table 3.

Table 3 compares performance efficiency achieved using hydrolysing destabilisation agent against polymeric coagulant when purifying water of a similar quality. In both cases the content of non-separable particles is the same, $C_{0} F=1.8 \mathrm{NTU}$. From this table (Polasek, 2011) the following is evident:

- The capability of the polymeric coagulant used to purify water is poor in comparison to aluminium sulphate. The concentration of the turbidity-producing non-aggregated particles of impurities remains high. This is evident from comparison of $C_{F} F_{C P E}=0.76 \mathrm{NTU}$ against $C_{F} F_{A l}=0.11 \mathrm{NTU}$, and the degree of destabilisation $\delta_{C P E}=0.58$ and $\delta_{A l}=0.94$ achieved by these respective systems. The filtration plant filtering the polymeric coagulant formed suspension is evidently not working efficiently, as the filtrate contains a high concentration of the separable suspension, $\Delta \mathrm{C}_{F-C P E}=$ $C_{F}-C_{F} F=1.03 \mathrm{NTU}$, in comparison to $\Delta \mathrm{C}_{F-A l}=0.06 \mathrm{NTU}$ attained in a different plant purifying similar quality of raw water using aluminium sulphate.

- The high concentration of the separable suspension in the filtrate $\Delta \mathrm{C}_{F-C P E}=1.03 \mathrm{NTU}$ is the result of inefficient backwashing, which is exacerbated by the use of polymeric coagulant.

The results presented in Table 4 (Polasek and Van Duuren, 1981) allow for comparison of the performance efficiency of different clarifiers installed at the same waterworks and purifying the same raw water. These clarifiers are the Pretreator, a radial flow type sludge blanket clarifier, the Clariflocculator, a radial flow sedimentation type clarifier and the HR (high rate) clarifier, vertical flow type sludge blanket clarifier. All clarifiers were operated to purify water to approximately the same total residual turbidity as measured in the clarifier outlet. As can be seen from the comparison of residual turbidity produced only by the non-separable particles $\left(C_{E} F\right)$, the performance efficiency of each system differs quite considerably. The best results were obtained with the HR clarifier system even though it operated at more than 10 times the hydraulic loading rate and with about 
one tenth of the retention time of the other clarifiers (Polasek and Van Duuren, 1981; Polasek and Mutl, 2005a, 2005b; Van Duuren, 1997). The reason for this is that the HR clarifier design is based on the principles of the high-rate clarification process. This incorporates the inline-high-density-suspension (IHDS) process in conjunction with the post-orthokinetic agglomeration (POA) process using organic flocculant aid (OFA). Under these processes the physical-chemical conditions of aggregate formation are optimised (Polasek and Mutl, 2005b; Van Duuren, 1997).

Both, the Pretreator and the Clariflocculator systems incorporated pre-flocculation which took place in a hydraulically operated around-end-baffle type flocculation plant having a retention time of about $20 \mathrm{~min}$. Discharge from the flocculation plant was divided to feed the Clariflocculator directly and the pretreator via a hydraulic jump (height of about $600 \mathrm{~mm}$ ). The hydraulic jump together with the sludge blanket improved the efficiency of the destabilisation process in the Pretreator system quite significantly in comparison to that of the Clariflocculator $\left(\mathrm{C}_{\mathrm{E}} \mathrm{F}_{\mathrm{P}}=1.5 \mathrm{NTU}\right.$ versus $\mathrm{C}_{\mathrm{E}} \mathrm{F}_{\mathrm{C}}=2.9 \mathrm{NTU}$ and $\delta_{\mathrm{P}}=0.972$ versus $\left.\delta_{\mathrm{C}}=0.946\right)$.

\section{CONCLUSIONS}

- The quantity of any pollutant in water is a sum of its two different particle size-fractions, namely, separable and non-separable particles. Reduction in the quantity of the non-separable particles is characterised by the degree of destabilisation $\delta$ and the efficiency of the development of aggregates is characterised by the degree of aggregation $\alpha_{A}$. The degree of destabilisation $\delta$ is proportionate to the purification process efficiency whereas the degree of aggregation $\alpha_{A}$ is proportionate to the aggregation and separation efficiency of the system.

- It was shown that the aggregation process produces separable aggregates and leaves a residual portion of impurities in the purified water which could not be destabilised and aggregated under the reaction conditions applied. The determination of the non-separable particles requires the removal of all kinetically unstable particles from the tested sample of water, some of which are very small $\left(d_{P} \approx 10^{-7} \mathrm{~m}\right)$. The removal of such small particles by plain sedimentation requires an impractically long time. Therefore, for the determination of the non-separable particles it is beneficial to replace sedimentation in a gravity field with sedimentation in a centrifugal field.

- The fraction of the non-separable particles remaining in the purified water determines the efficiency of the destabilisation process attained by the system under the applied reaction conditions, as well as the overall process efficiency of the works.

- Based on definitions of both the collision efficiency factor $\alpha_{p}$ in the Smoluchovski Eq. for perikinetic coagulation and the degree of destabilisation $\delta$, it is evident that the value of $\delta$ can be used to enumerate the factor $\alpha_{p}$. Similarly, the value of degree of aggregation $\alpha_{A}$ can be used to enumerate factor $\alpha_{O}$ in the Smoluchovki Eq. for orthokinetic coagulation.

- The degree of destabilisation $\delta$ and the degree of aggregation $\alpha_{A}$ are the criteria determining the efficiency of the partial processes of water purification. They are simple tools enabling evaluation and determination of the optimised chemical-physical conditions of the purification process, selection of the most suitable unit operations and arrangement of the entire waterworks. Furthermore, they can be used for the identification of bottlenecks in water purification process.

- The conditions of centrifugation can be calculated or experimentally established for any type of centrifuge and its speed of rotation, as shown in Table 3.

- The degree of destabilisation is an indispensible tool for the evaluation of the performance efficiency of waterworks, as is evident from Tables 3 and 4.

\section{REFERENCES}

HEINRICH J, ILAVSKY J, KOSSACZKY E, LODES A and SUROVY J (1970) Chemical engineering lessons and exercises (in Slovak). Alfa Publisher, Bratislava.

HEREIT F, MUTL S and VAGNER V (1977) Evaluation of waterworks performance (in Czech). J. Vodni hospodarstvi 4-B (27) 80-86.

HEREIT F, MUTL S and VÁGNER V (1980) Formation of separable suspensions and methods of its assessment. Aqua 595.

MUTL S and POLASEK P (2002) Particle sizes and particle sizefraction distribution in water treatment. Poster presentation: IWA Specialist Conf. Particle Separation, April 2002, Melbourne, Australia.

POLASEK P (1980) Methods and testing procedures for monitoring and evaluating waterworks performance. Proc. Int. Conf. SAFIL, October 1980, Johannesburg.

POLASEK P (2011) Functional status quo assessment report on Enqcobo WTW, Chris Hani District Municipality. September 2011. Development Bank of Southern Africa, Pretoria.

POLASEK P and MUTL S (2002) Cationic polymers in water treatment: Part 1: Treatability of water with cationic polymers, Part 2: Filtration of water treated by cationic polymers. Water $S A 28$ (1) 69-88.

POLASEK P and MUTL S (2005a) Acceleration of gravity separation processes. J. Filtration 5 (1) 33-39.

POLASEK P and MUTL S (2005b) High rate clarification technology. Proc. IWA Specialised Conference: Particle Separation 2005: Sustainable and Innovative Technologies to Meet MDGs, 1-3 June 2005, Seoul, Korea.

POLASEK P and VAN DUUREN FA (1981) The performance results from the operation of high rate clarifiers at Bethlehem Municipal Waterworks. Water Industry '81, June 1981, Brighton.

SMOLUCHOWSKI M (1916) Drei vortrage uber diffusion, brownische bewegung und koagulation von kolloidteilchen. Z. Phys. 17 557-571.

SMOLUCHOWSKI M (1917) Versuch einer mathematischen theorie der koagulationskinetik kolloider losungen. Z. Phys. Chem. 92 129-168.

VAN DUUREN FA (1997) Water Purification Works Design. WRC Report No. TT 92/97. Water Research Commission, Pretoria. 